\title{
Laparoscopic adjustable gastric banding: Technique and results
}

\author{
Oktay Banlı, ${ }^{1}$ Hasan Altun, ${ }^{2}$ Ergin Aslan, ${ }^{1}$ Mesut Sipahi, ${ }^{1}$ \\ Hasan Börekçi,' Kasım Çağlayan' ${ }^{1}$ \\ 'Department of General Surgery, Bozok University Faculty of Medicine, Yozgat, Turkey \\ ${ }^{2}$ Department of Obesity and Metabolic Surgery, Liv Hospital, Istanbul, Turkey
}

\begin{abstract}
Prevalence of obesity is increasing worldwide and bariatric surgery is the most effective surgical therapy for obesity. Laparoscopic adjustable gastric banding (LABG) is one of the most common bariatric procedures in the world. Popularity is high among surgeons due to shorter learning curve and lower early complication rate. There are 2 insertion techniques for LABG: the perigastric technique and pars flaccida technique. Pars flaccida approach evolved due to high complication rate of perigastric technique. Most common complications of LABG are pouch dilation, band slippage, band migration, and port complications. Although LABG is an effective weight loss procedure, weight loss is slower compared with other bariatric procedures, such as gastric sleeve or bypass. It results in significant improvement in co-morbidities, including diabetes, hypertension, hyperlipidemia, and sleep apnea, and significant improvement in quality of life is seen. Despite the high safety profile of LABG in short term, it has a high complication rate in long term. Patients who are candidates for LABG must understand that intensive follow-up, compliance, and motivation are necessary for good results and outcomes.
\end{abstract}

Keywords: Bariatric surgery; laparoscopic adjustable gastric banding; obesity

\section{Introduction}

Obesity is increasing worldwide. In addition to causing many co-morbidities like hypertension, diabetes and sleep apnea, it also causes an increased burden to the healthcare system. Nonsurgical treatment methods of obesity like diet and exercise may achieve only a 5-10\% weight loss. ${ }^{[1,2]}$

Laparoscopic adjustable gastric banding (LABG) represents one of the most frequently performed bariatric operations in morbidly obese patients. Popularity of LABG is high among surgeons due to shorter learning curve and lower early complication rate. Despite its improved early safety compared with other bariatric procedures, it may cause unique complications and require a specific procedure for assessment and management.

Gastric band is losing its popularity in Europe and gaining popularity in the United States. ${ }^{[3]}$ Food and Drug Administration in the United States approved LAP-BAND for a body mass index greater than 30 in 2001. Until that time, the use of LABG was increasing in the United States. 


\section{History}

Vertical banded gastroplasty is a pioneer of the gastric band, being the first restrictive bariatric operation. ${ }^{[4]}$ Surgical restriction of intake by means of a nonadjustable gastric band was started in the 1980s. ${ }^{[5]}$ First step was nonadjustable gastric band and replaced gastroplasty. Gastric band tried to eliminate the disadvantages of vertical banded gastroplasty by placing silicon band around the stomach.

First adjustable gastric band was introduced by Kuzmak. ${ }^{[6]}$ Belachew was the first to perform laparoscopic adjustable gastric band (LABG) operation in 1993..$^{[7,8]}$ The techniqueof Belachew was the perigastric technique. ${ }^{[5]}$ After laparoscopic operations, LABG gained popularity around the world as a first-choice surgical therapy for morbid obesity.

\section{Surgical Technique}

Main parts of the gastric band are silicone band, access port and connecting tube. Principle of this technique is to create an adjustable opening and a small gastric pouch to limit food consumption and induce early satiety. There are two different techniques in adjustable gastric band placement including perigastric technique and pars flaccida technique.

\section{Perigastric Technique}

Belachew's original technique for band placement was called perigastric technique, which involves creating a window at the lesser curvature of the stomach $3 \mathrm{~cm}$ below

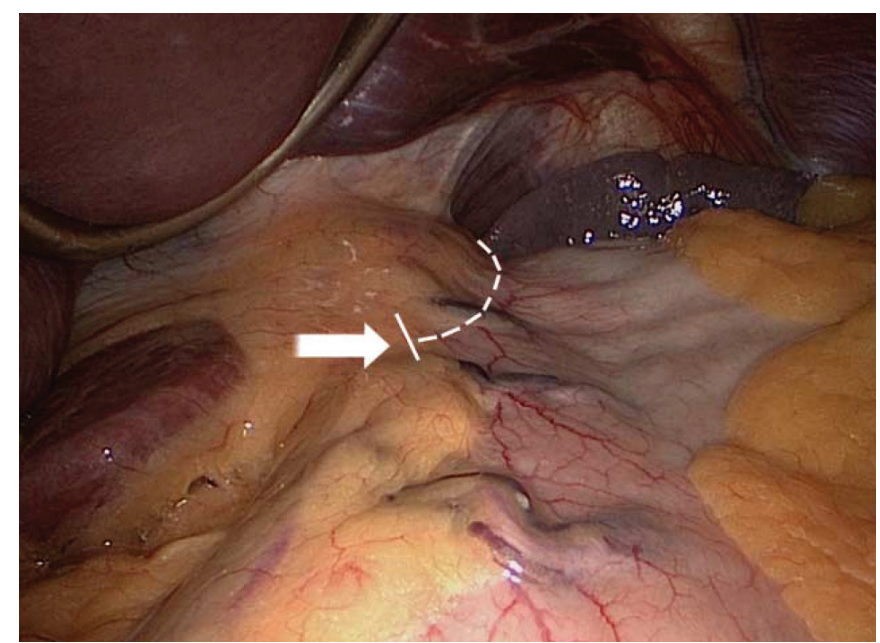

Figure 1. Perigastric technique. Dissection is performed near the gastric wall $3 \mathrm{~cm}$ below the gastroesophageal junction. White arrow: entry point, dotted line: route of retrogastric tunnel. the gastro-esophageal junction. ${ }^{[5]}$ The window is created medial to the neurovascular bundle and a tunnel is created below the gastric wall and extended to the angle of His with blunt dissection (Figure 1). Band is introduced into the abdomen through a trocar with mounting straight on a grasper. The band is pulled through this tunnel with a grasper to create $15-30 \mathrm{~mL}$ gastric pouch above the band. Then, a calibration bougie is inserted orally into the stomach and inflated with $25 \mathrm{cc}$ saline. The bougie is withdrawn to the esophago gastric junction. Band is closed to create gastric pouch below the balloon of the bougie. In order to avoid band slippage, gastric plication is created by putting, generally, three to five anterior sutures between the greater curvature and gastric pouch. Connecting tube is pulled outside the abdomen through a trocar and is connected and linked to the port after trocar removal. The port is fixed to the abdominal fascia with non-absorbable sutures.

\section{Pars Flaccida Technique}

The perigastric technique has evolved into the pars flaccida technique due to the complications of perigastric technique. ${ }^{[9,10]}$ Since this technique has low complexity in dissection maneuvers, it is the most commonly utilized and generally recommended technique. The dissection begins near the angle of His. The thin area of gastrohepatic ligament (pars flaccida) over the caudate lobe is divided. After the identification of right crus, peritoneum at the border of the crus is incised. The grasper is passed behind the gastro-esophageal junction to the angle of His (Figure 2). The band is introduced into the abdomen through the

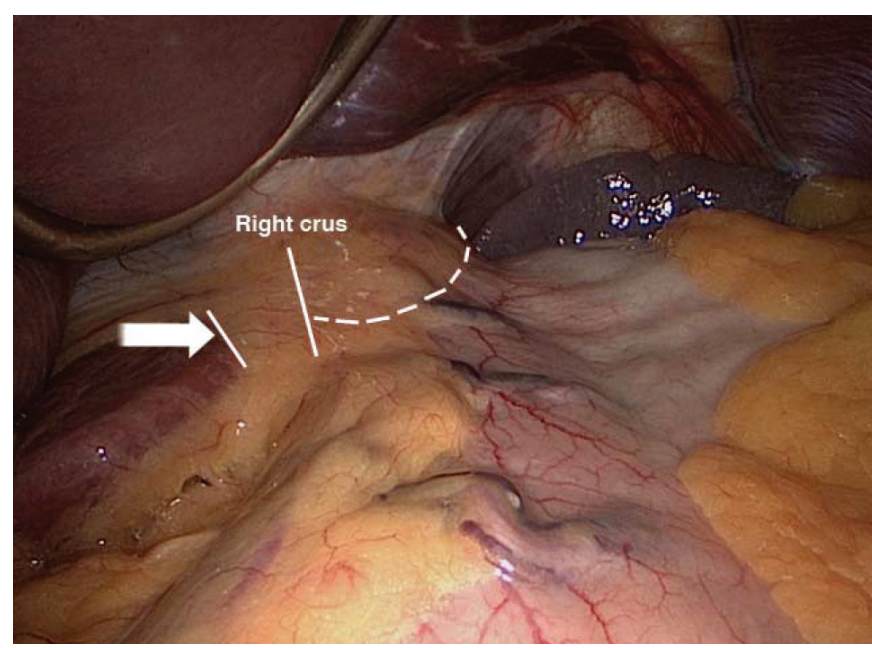

Figure 2. Pars flaccida technique. After dividing gastrohepatic ligament, the dissection continues anterior to the right crus. Opening pars. White arrow: entry point, dotted line: route of retrogastric tunnel. 
port. The end of the band is grasped with the grasper and passed through the created tunnel. A calibration bougie is passed to the stomach through oral route. The balloon at the end of the bougie is filled with $15 \mathrm{cc}$ saline.

The bougie is withdrawn to the esophagogastric junction. Band is closed to create gastric pouch below the balloon of the bougie. In order to avoid band slippage, gastric plication is created by putting, generally, three to five anterior sutures between the anterior gastric wall and gastric pouch. Connecting tube is pulled outside the abdomen through a trocar and is connected and linked to the port after trocar removal. The port is fixed to the abdominal fascia with non-absorbable sutures.

Adjustment: At the time of the operation, no fluid is injected into the band, and it is left empty. First filling is performed after 4-6 weeks based on the patient's rate of weight loss and food-fluid tolerance. If the patient has insufficient weight loss (less than $0.5 \mathrm{~kg}$ per week) and insufficient restriction of solid food, fluid is injected to the port. If the patient has obstructive symptoms (vomiting, heart burn), the fluid must be removed from the port. Generally, the port can be accessed by palpation. If it cannot be accessed by palpation, fluoroscopy should be used. After the adjustment, the patient should be able to drink water comfortably. The patient must be followed up every 4-8 weeks during the first postoperative year.

\section{Results}

\section{Complications}

Safety is the major perioperative advantage of LABG. Despite the initial success and safety of LABG, more recent studies show rising long-term complications. ${ }^{[11]}$ It has been suggested that each year the band is increasing the complication rate 3 to $4 \% .{ }^{[12]}$ Pouch dilation, band slippage, band migration, and port problems represent the complications most commonly associated with LABG. ${ }^{[13]}$

Pouch dilation is a different entity from slippage. There is a concentric dilation and the band is seen in its normal axis in radiography. Tight band or overeating may cause this complication. Endoscopy reveals concentric dilation and there is a narrow segment on barium radiography. Band fluid removal is usually curative. ${ }^{[1]}$ Over a period of time, resolution of the dilation occurs in most patients.

Band slippage or gastric prolapse occur when a portion of the gastric wall herniates under the band and there is a rotation of the band. O'Brien has reported band slippage rate of less than $5 \% .{ }^{[14]}$ Perigastric technique causes more frequent band slippage. Anterior or posterior gastric wall prolapse may be seen. Posterior slippage is seen more frequently in the perigastric technique. However, it is less frequent now since the par flaccida technique is adopted instead of the perigastric technique. Band slippage generally requires immediate operation. Some surgeons prefer repositioning the band. However, recurrence is common with this approach. ${ }^{[11]}$ Replacement of the band with a newly created tunnel is an alternative method. Conversion to other bariatric procedures as first stage or second stage is an acceptable alternative therapy.

Band migration is the erosion of the gastric wall with the band and migration of the band into the gastric lumen. The incidence ranges from 0 to $5.8 \% .{ }^{[1]}$ The incidence is higher in long standing bands. Most patients with band migration are asymptomatic and weight regain may be the only symptom. The etiology includes gastric wall injury during placement and tight fixation around the band. Endoscopy is the diagnostic tool for suspicious cases. The treatment is laparoscopic removal of the band and repairment of the gastric wall. ${ }^{[15]}$ Endoscopic removal with specific devices is an alternative therapy. ${ }^{[15,16]}$

Port complications are seen more frequently and include disconnection of the tube, skin ulceration, and port-site infection. ${ }^{[13]}$ Port-site infections are the most frequent complications and can be classified as early and late. ${ }^{[17]}$ Early infection occurs in the immediate postoperative period. Therapy is oral or intravenous antibiotic. When the infection doesn't heal, the port should be removed and a new port placed as a second stage. Late infections generally occur as an ascending infection due to band migration. Therapy of this later infection is the removal of the band. ${ }^{[13]}$ Skin ulceration is a rare complication of the port, occurring, generally, as a result of weight loss and loses of subcutaneous fatty tissue. ${ }^{[17]}$ Using a low profile port and inserting the port deeply may prevent this complication.

\section{Outcomes}

Weight loss: LABG is an effective weight-loss procedure with the majority of patients losing between 44 and 55\% of their excess body weight. ${ }^{[18]}$ This technique results in a slower weight loss compared to other bariatric procedures like gastric bypass and sleeve gastrectomy. ${ }^{[19]}$

LABG results in less weight loss in the first 6 monthsand starts to reach approximately 50 to $65 \%$ in $2-3$ years. ${ }^{[20-23]}$ 
There are gradual weight losses and many factors affecting weight loss after LABG. Close follow-up visits with band adjustment are necessary for a successful weight loss.

Co-morbidities: One of the most important outcome measures of bariatric surgery is the resolution of comorbid conditions. Unlike the metabolic effects of other bariatric procedures, the remission or improvement of comorbidities after LABG is believed to be due to the direct effects of weight loss. Weight loss can improve type 2 diabetes, hypertension, gastro esophageal reflux, hyperlipidemia, sleep apnea, polycystic ovarian syndromes, arthritis, and overall quality of life. O'Brien and Frigg have shown that after LABG, patients experience $94 \%$ resolution of sleep apnea, 55-75\% resolution of diabetes, 55-58\% resolution of hypertension, and $74 \%$ resolution of hyperlipidemia. ${ }^{[24,25]}$ In a meta-analysis, Buchwald has shown $76.8 \%$ resolution of diabetes, $71.1 \%$ improvement in hyperlipidemia, and $71.5 \%$ resolution or improvement in hypertension. ${ }^{[26]}$

Quality of life: There are some methods to measure quality of life (QoL) after bariatric surgery. Most commonly used one is the Short Form-36 Health Survey (SF-36). There is increase in QoL after LAP in short term. ${ }^{[27-29]}$ Weiner has also shownstable improvement in the long-term in QoL in patients undergoing LAGB. ${ }^{[22]}$

\section{Discussion}

Surgical technique in LABG has undergone some evolution to become an effective option for patients. The most important modification in the technique has been the introduction of an alternative pathway. Since the perigastric technique has resulted in frequent complications, pars flaccida technique has been developed. The most common problem with the perigastric technique was gastric prolapse. ${ }^{[10]}$ O'Brien demonstrated significant decrease in complication of gastric prolapse when the surgical technique was modified to pars flaccida. ${ }^{[9,30]}$ The change to pars flaccida technique led to lower incidence of band removal.

There are some advantages of LABG over other bariatric procedures. The operation time is short, it is reversible, and stoma size is adjustable. It has low mortality rate and excellent safety profile for intraoperative and early postoperative complications. ${ }^{[11]}$ Patients may undergo other bariatric procedures if necessary. LABG may be an option for patients who want less invasive bariatric procedure.
However, it comes with risk of possible reoperation rate for band slippage, erosion, port problems, and band migration. ${ }^{[30]}$ There is a steep decrease in LABG with a concomitant steep increase in sleeve gastrectomy as a decrease in weight loss efficacy and an increase in longterm complications can be seen in LABG. ${ }^{[3]}$ Patients, who are candidates for LABG, must understand that intensive follow up, compliance and motivation are necessary for good results and outcomes.

\section{References}

1. O'Brien PE, McPhail T, Chaston TB, Dixon JB. Systematic review of medium-term weight loss after bariatric operations. Obes Surg 2006;16:1032-40.

2. Fisher BL, Schauer P. Medical and surgical options in the treatment of severe obesity. Am J Surg 2002;184:9S-16S.

3. Buchwald H, Oien DM. Metabolic/bariatric surgery worldwide 2011. Obes Surg 2013;23:427-36.

4. Mason EE. Vertical banded gastroplasty for obesity. Arch Surg 1982;117:701-6.

5. Belachew M, Jacqet P, Lardinois F, Karler C. Vertical Banded Gastroplasty vs Adjustable Silicone Gastric Banding in the Treatment of Morbid Obesity: a Preliminary Report. Obes Surg 1993;3:275-8.

6. Kuzmak LI, Yap IS, McGuire L, Dixon JS, Young MP. Surgery for morbid obesity. Using an inflatable gastric band. AORN J 1990;51:1307-24.

7. Belachew $M$, Legrand MJ, Defechereux TH, Burtheret MP, Jacquet $\mathrm{N}$. Laparoscopic adjustable silicone gastric banding in the treatment of morbid obesity. A preliminary report. Surg Endosc 1994;8:1354-6.

8. Belachew M, Legrand MJ, Vincent V. History of Lap-Band: from dream to reality. Obes Surg 2001;11:297-302.

9. O'Brien PE, Dixon JB, Laurie C, Anderson M. A prospective randomized trial of placement of the laparoscopic adjustable gastric band: comparison of the perigastric and pars flaccida pathways. Obes Surg 2005;15:820-6.

10. Di Lorenzo N, Furbetta F, Favretti F, Segato G, De Luca M, Micheletto $\mathrm{G}$, et al. Laparoscopic adjustable gastric banding via pars flaccida versus perigastric positioning: technique, complications, and results in 2,549 patients. Surg Endosc 2010;24:1519-23.

11. Snow JM, Severson PA. Complications of adjustable gastric banding. Surg Clin North Am 2011;91:1249-64.

12. Biertho L, Steffen R, Ricklin T, Horber FF, Pomp A, Inabnet WB, et al. Laparoscopic gastric bypass versus laparoscopic adjustable gastric banding: a comparative study of 1,200 cases. J Am Coll Surg 2003;197:536-44.

13. Eid I, Birch DW, Sharma AM, Sherman V, Karmali S. Complications associated with adjustable gastric banding for morbid obesity: a surgeon's guides. Can J Surg 2011;54:61-6.

14. O'Brien PE, Dixon JB. Weight loss and early and late complications-the international experience. Am J Surg 
2002;184:42S-45S.

15. Cherian PT, Goussous G, Ashori F, Sigurdsson A. Band erosion after laparoscopic gastric banding: a retrospective analysis of 865 patients over 5 years. Surg Endosc 2010;24:2031-8.

16. Neto MP, Ramos AC, Campos JM, Murakami AH, Falcão M, Moura EH, et al. Endoscopic removal of eroded adjustable gastric band: lessons learned after 5 years and 78 cases. Surg Obes Relat Dis 2010;6:423-7.

17. Susmallian S, Ezri T, Elis M, Charuzi I. Access-port complications after laparoscopic gastric banding. Obes Surg 2003;13:128-31.

18. Brethauer SA, Chand B, Schauer PR. Risks and benefits of bariatric surgery: current evidence. Cleve Clin J Med 2006;73:993-1007.

19. Chapman AE, Kiroff G, Game P, Foster B, O'Brien P, Ham J, et al. Laparoscopic adjustable gastric banding in the treatment of obesity: a systematic literature review. Surgery 2004;135:326-51.

20. O'Brien PE, Dixon JB. Lap-band: outcomes and results. J Laparoendosc Adv Surg Tech A 2003;13:265-70.

21. O'Brien PE, Dixon JB, Brown W, Schachter LM, Chapman L, Burn AJ, et al. The laparoscopic adjustable gastric band (Lap-Band): a prospective study of medium-term effects on weight, health and quality of life. Obes Surg 2002;12:652-60.

22. Weiner R, Blanco-Engert R, Weiner S, Matkowitz R, Schaefer
L, Pomhoff I. Outcome after laparoscopic adjustable gastric banding - 8 years experience. Obes Surg 2003;13:427-34.

23. Vertruyen M. Experience with Lap-band System up to 7 years. Obes Surg 2002;12:569-72.

24. Dixon JB, O'Brien PE. Changes in comorbidities and improvements in quality of life after LAP-BAND placement. Am J Surg 2002; 184:51S-54S.

25. Frigg A, Peterli R, Peters T, Ackermann C, Tondelli P. Reduction in co-morbidities 4 years after laparoscopic adjustable gastric banding. Obes Surg 2004;14:216-23.

26. Buchwald $H$, Avidor $Y$, Braunwald E, Jensen MD, Pories W, Fahrbach K, et al. Bariatric surgery: a systematic review and meta-analysis. JAMA 2004;292:1724-37.

27. Brancatisano A, Wahlroos S, Brancatisano R. Improvement in comorbid illness after placement of the Swedish Adjustable Gastric Band. Surg Obes Relat Dis 2008;4:S39-46.

28. Ahroni JH, Montgomery KF, Watkins BM. Laparoscopic adjustable gastric banding: weight loss, co-morbidities, medication usage and quality of life at one year. Obes Surg 2005; 15:641-7.

29. Rubenstein RB. Laparoscopic adjustable gastric banding at a U.S. center with up to 3-year follow-up. Obes Surg 2002;12:380-4.

30. McBride CL, Kothari V. Evolution of laparoscopic adjustable gastric banding. Surg Clin North Am 2011;91:1239-47. 